\title{
PROBLEMAS EN LA INTEGRACIÓN SENSORIAL EN NIÑOS HUÉRFANOS, INSTITUCIONALIZADOS Y ADOPTADOS: UNA REVISIÓN EXPLORATORIA
}

SENSORY INTEGRATION PROBLEMS IN ORPHANED, INSTITUTIONALIZED AND

ADOPTED CHILDREN: A SCOPING REVIEW

\section{Ana Muñoz Meraํ․ Sabina Barrios Fernández ${ }^{2}$}

\begin{abstract}
RESUMEN
Objetivo: conocer el estado actual del conocimiento sobre de problemas en el procesamiento sensorial en niños huérfanos, institucionalizados y adoptados. Metodología: revisión en la base de datos SCOPUS y en el buscador PubMed. Resultados: 12 trabajos que cumplen con los criterios de inclusión-exclusión. De ellos, 7 ilustran los problemas de salud y disfunciones ocupacionales de los niños que residen en instituciones y otros 5, de los niños adoptados. Conclusiones: A la luz de los estudios encontrados, parece ser que los niños institucionalizados, huérfanos y adoptados podrían presentan dificultades relacionadas con problemas en la Integración Sensorial.
\end{abstract}

\section{PALABRAS CLAVE}

Niño institucionalizado, niño adoptado, sensación, desempeño psicomotor.

\begin{abstract}
Aim: examining the current state of knowledge about the problems in sensory processing in orphaned, institutionalized and adopted children. Methods: a review in the SCOPUS database and in the PubMed search engine was conducted. Results: 12 studies met the inclusion-exclusion criteria. 7 illustrated health problems and occupational dysfunctions of children residing in institutions. The other 5 examined problems in adopted children. Conclusions: to our best knowledge, institutionalized, orphaned and adopted children should present problems related to sensory integration.
\end{abstract}

\section{KEYWORDS}

Orphaned child, adopted child, sensation, Psychomotor Performance.

Recibido: 10 Septiembre 2019

Aceptado: 29 Noviembre 2019

1 Graduada en Terapia Ocupacional Universidad de Extremadura. Correspondencia: Calle Luis Buñuel n ${ }^{\circ} 2 . \mathrm{CP}_{10} 55 \mathrm{O}$. Aliseda (Cáceres). Email anamuozmera@gmail.com Teléfono: 665447066

2 Diplomada en Terapia Ocupacional en la Universidad de Extremadura. Doctora en Psicología por la Universidad de Extremadura. Profesora en el grado en Terapia Ocupacional de la Universidad de Extremadura. Dirección de correspondencia. Calle Luis Buñuel no $2 . \mathrm{CP}_{10550}$ Aliseda (Cáceres). Email sabinabarrios@unex.es. Teléfono: 665447066 


\section{INTRODUCCIÓN}

\section{La importancia de los primeros años}

La infancia es un periodo durante el cual se conquistan numerosos hitos. No obstante, es importante recordar que el desarrollo humano tiene una serie de características (Papalia, Martorrell, \& Duskin Feldman, 2013): es vitalicio, depende de los entornos y los contextos, es multidimensional y multidireccional y es flexible, plástico. De esta forma, entorno y contexto son dos aspectos determinantes para el desarrollo ocupacional del niño en particular, y del ser humano, en general (Anaby, Law, Teplicky, \& Turner, 2015; Domínguez Jiménez \& Calvo Arenillas, 2015). La Asociación Americana de Terapia Ocupacional (AOTA, 2014) define los entornos (físico y social) como los ambientes en los cuales se realizan las ocupaciones, mientras que los contextos (cultural, personal, temporal y virtual) serían una serie de condiciones que influyen en el desempeño ocupacional. Según Rajmil (2013) un entorno empobrecido o poco estimulante puede influir tanto en el desarroIlo como en la calidad de niño. También son de vital importancia, como indica Chamorro Noceda (2012) las relaciones, cuidados y/o vínculos de los niños con sus familiares / cuidadores principales, que deben ser suficientemente buenas para evitar problemas en el desarrollo. Así, tanto el lugar como las relaciones que se producen en los primeros años pueden condicionar el desarrollo del niño, ya que éste va a ser el resultado de la interacción entre sus capacidades potenciales y su entorno (Domenech, 2001). De esta forma, un cerebro en maduración presenta una mayorvulnerabilidad a las condiciones adversas del medio pudiendo producirse un trastorno del desarrollo por circunstantes sociales poco favorecedoras, incluyendo situaciones de negligencia, maltrato o abusos (Federación Estatal de Asociaciones de Profesionales de Atención Temprana, 2005).

\section{Caracterización de los niños huérfanos, institucionalizados y adoptados}

Según UNICEF (2015), un niño huérfano es aquel que ha perdido a uno (huérfano de padre o madre) o ambos progenitores (huérfano doble). Según dicha organización, habría unos 140 millones de huérfanos en el mundo, de los cuales 15 millones habrían perdido a sus dos padres. La mayoría de ellos estaría al cuidado de un familiar, pero aún así se estima que casi 2,5 millones de niños vivirían en entornos de cuidado institucional (Petrowski, Cappa, \& Gross, 2017). Tomando como referencia el año 2017, unos 14.000 niños estarían bajo la tutela del estado español, mientras que esta cifra sería de 206 niños por cada 100.00o en Chile. Durante el mismo año se produjeron en España 68o adopciones, mientras que en Chile se registraron 428, con una tendencia de cifras a la baja en ambos países (Observatorio de la Infancia, 2018; Observatorio para la Confianza, 2019; Servicio Nacional de Menores, 2019).

Según Juffer, van Ijzendoorn, \& Palacios (2011) y VegaArce \& Nuñez-Ulloa, (2017), muchos de los niños que pasan parte de su vida en centros residenciales tutelados por el estado han pasado por situaciones sociales graves que han impactado en su desarrollo y en su salud. Cermak (2009) indica que vida en un orfanato se caracteriza porque hay múltiples cuidadores que se turnan para cuidar a los niños, por lo que se da una alta ratio niños-cuidador y además el entorno puede estar configurado de forma que pueden tener menor oportunidad de movimiento y juegos. Hernándezmuela, Mulas, Meneses, \& Roselló (2003) han observado distintas enfermedades y/o patologías en niños huérfanos, institucionalizados y adoptados: trastornos nutricionales, raquitismo, anemia ferropénica, dificultades en el desarrollo psicomotor, alteraciones en comunicación y lenguaje, trastornos de la conducta, trastorno por déficit de atención e hiperactividad, problemas emocionales y alteraciones psicológicas y psiquiátricas (Wiik et al., 2011).

\section{Teoría de Integración Sensorial}

La Integración Sensorial (IS) es un proceso neurobiológico que consiste en la adecuada organización de las sensaciones que provienen de los sentidos en el Sistema Nervioso Central (SNC) para la elaboración de respuestas adaptativas en la vida diaria (Ayres \& Robbins, 2008). Además de la teoría, Ayres desarrolló el marco clínico teórico-clínico de intervención específico de Terapia Ocupacional desde el cual, en un ambiente seguro, se produce a través del juego configurado mediante retos alcanzables que producen respuestas adaptativas que permiten el aprendizaje del niño (Ayres, 1972). Así, un trastorno del procesamiento sensorial (TSP) resultaría de un problema relacionado con una inadecuada 
integración de las sensaciones en el cerebro, pudiendo producirse problemas en la modulación, discriminación o dificultades motoras de base sensorial (Miller, Anzalone, Lane, Cermak, \& Osten, 2007). Las repercusiones de dicho TPS podrían derivar en dificultades en la vida diaria y participación, incluyendo problemas de aprendizaje, conducta, sociales, problemas emocionales (de autoestima y autocontrol), de coordinación motriz o de atención. Por todo ello, la detección precoz de un TPS resulta primordial para prevenir futuras disfunciones ocupacionales (del Moral Orro, Pastor Montaño, \& Sanz Valer, 2013).

Se plantea que algunas de estas disfunciones ocupacionales que muestran los niños huérfanos, institucionalizados y adoptados pudieran ser debidas a dificultades en la IS, por lo que se plantea como objetivo de este trabajo conocer y analizar el estado actual de conocimiento en relación a la IS en estos niños.

\section{MÉTOdo}

Se realizó una búsqueda en la base de datos SCOPUS y en el buscador PubMed. Se incluyen las palabras clave "child, orphaned", "child, institutionalized", "child, adopted", "sensory proccesing" $y$ "problems". Se utilizaron los operadores booleanos lógicos AND y OR, además del correspondiente uso de paréntesis y comillas.

Con respecto a los criterios de inclusión-exclusión, se han incluido estudios que han sido publicados entre 2013 y 2019, se han realizado en niños y adolescentes de hasta 18 años huérfanos, institucionalizados o adoptados, si son publicaciones con acceso abierto y están escritas en inglés, español o francés. Por otro lado, se han excluido artículos en los que el texto completo no se encontraba disponible, estaban escritos en un idioma distinto a los mencionados o si no cumplían los criterios previamente mencionados. En la figura 1 se ilustra el diagrama de flujo con el proceso de selección de los trabajos en función de los criterios expuestos.
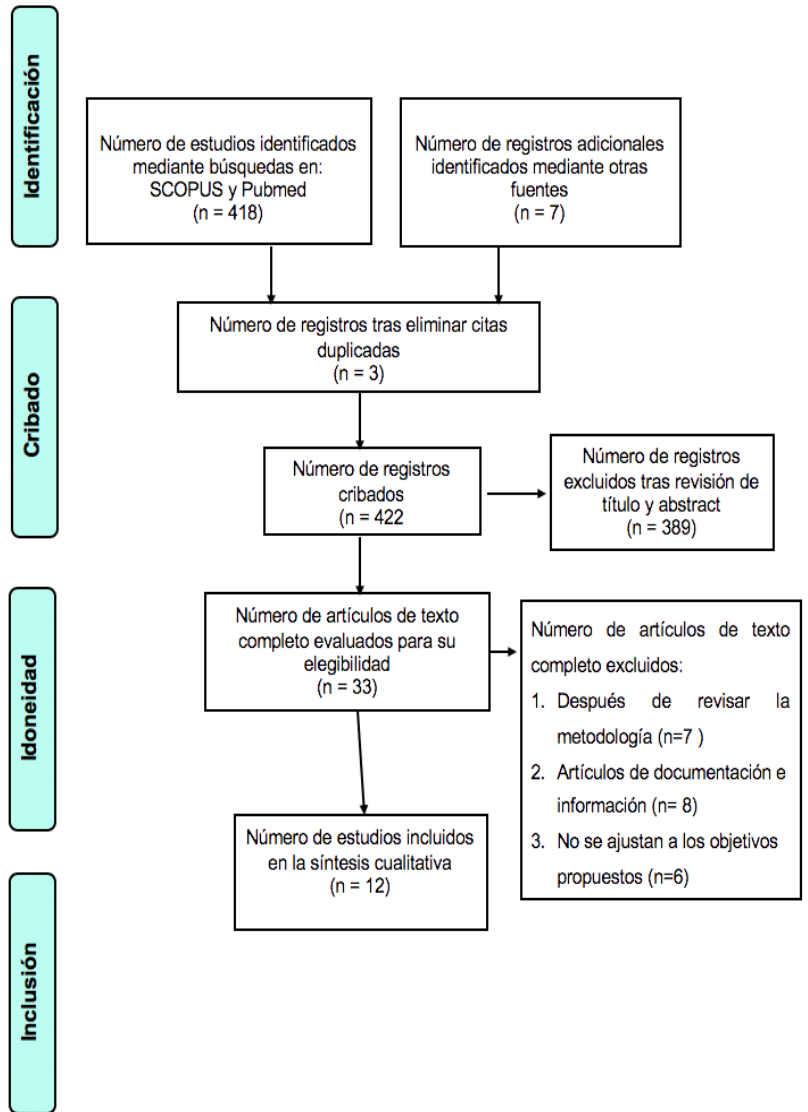

FIGURA 1. DIAGRAMA DE FLUJO. (ELABORACIÓN PROPIA)

\section{ReSULTADOS}

En la tabla 1 se recogen los trabajos que muestran los déficits de los niños institucionalizados, que suelen presentar más problemas de hiperactividad, agresión o conductas delincuentes, peor rendimiento académico, depresión, ansiedad, quejas somáticas o retraimiento social X. 


\begin{tabular}{|c|c|c|c|c|c|}
\hline Autor, año y título & Objetivo & Muestra & $\begin{array}{l}\text { Instrumentos } \\
\text { de evaluación }\end{array}$ & Procedimiento & Resultados \\
\hline $\begin{array}{l}\text { McDermott et al., (2013) } \\
\text { Psychosocial depriva- } \\
\text { tion, executive functions, } \\
\text { and the emergence of } \\
\text { socio-emotional behavior } \\
\text { problems. }\end{array}$ & $\begin{array}{l}\text { CAUG se desempeñarían peor } \\
\text { que NIG en las medidas de } \\
\text { función ejecutiva de control } \\
\text { inhibitorio y monitoreo de la } \\
\text { respuesta. } \\
\text { FCG se desempeñarían en un } \\
\text { nivel intermedio en compara- } \\
\text { ción con el CAUG y el NIG. }\end{array}$ & $\begin{array}{l}49 \text { niños CAUG, } \\
54 \text { niños FCG y } 47 \\
\text { NIG. } \\
\text { Edad media de } 8 \\
\text { años. }\end{array}$ & $\begin{array}{l}\text { Tarea de los } \\
\text { flankos. } \\
\text { Electroencefalo- } \\
\text { grama. } \\
\text { HBQ. } \\
\text { SSRS. } \\
\text { WISC-IV. }\end{array}$ & $\begin{array}{l}\text { Se evalúa a los niños de } \\
\text { la muestra para examinar } \\
\text { los efectos que tiene la } \\
\text { adversidad temprana, los } \\
\text { efectos potenciales de la } \\
\text { intervención en la FE y el } \\
\text { papel de las habilidades } \\
\text { de la FE en los resultados } \\
\text { socioemocionales. }\end{array}$ & $\begin{array}{l}\text { CAUG y FCG fueron menos } \\
\text { precisos, tuvieron tiempos de } \\
\text { reacción más lentos que el NIG. } \\
\text { CAUG y FCG presentan signi- } \\
\text { ficativamente más comporta- } \\
\text { mientos de externalización que } \\
\text { el NIG. } \\
\text { FCG y CAUG presentan com- } \\
\text { petencias académicas menores } \\
\text { que el NIG. }\end{array}$ \\
\hline $\begin{array}{l}\text { (Levin, Zeanah, Fox, \& } \\
\text { Nelson, (2014) } \\
\text { Motor Outcomes in Chil- } \\
\text { dren Exposed to Early } \\
\text { Psychosocial Deprivation. }\end{array}$ & $\begin{array}{l}\text { Determinar el efecto de la } \\
\text { privación psicosocial tempra- } \\
\text { na sobre el desarrollo motory } \\
\text { evaluar el impacto de la inter- } \\
\text { vención de acogida temporal } \\
\text { en la mejora del desarrollo } \\
\text { motor y la asociación entre los } \\
\text { resultados motores y cogniti- } \\
\text { vos en niños con anteceden- } \\
\text { tes de atención institucional. }\end{array}$ & $\begin{array}{l}47 \text { niños CAUG, } \\
50 \text { niños FCG y } 60 \\
\text { NIG. } \\
\text { Edad media de } 8 \\
\text { años. }\end{array}$ & BOT2-SF. & $\begin{array}{l}\text { Se evalúa la competencia } \\
\text { motriz de los niños. }\end{array}$ & $\begin{array}{l}\text { Los resultados en el BOT2-SF } \\
\text { fueron significativamente mejo- } \\
\text { res en el NIG que en el EIG. } \\
\text { No hubo diferencias significati- } \\
\text { vas entre el CAUG y el FCG. } \\
\text { NIG tuvo una puntuación equi- } \\
\text { valente a su edad (8 años). } \\
\text { SI tuvo una puntuación equiva- } \\
\text { lente a edad de } 6,5 \text { años. }\end{array}$ \\
\hline $\begin{array}{l}\text { Mbiya Muadi et al., } \\
\text { (2014) } \\
\text { Attachement et déve- } \\
\text { loppement des enfants } \\
\text { abandonnés vivant en } \\
\text { institution résidentielle à } \\
\text { Kinshasa. }\end{array}$ & $\begin{array}{l}\text { Evaluar el efecto de la calidad } \\
\text { del apego en el desarrollo } \\
\text { cognitivo y físico de los niños } \\
\text { colocados en instituciones } \\
\text { para niños abandonados en } \\
\text { Kinshasa. }\end{array}$ & $\begin{array}{l}84 \text { niños: } 42 \text { niños } \\
\text { en CAUG y } 42 \text { NIG. } \\
\text { De } 4 \text { a } 7 \text { años. }\end{array}$ & $\begin{array}{l}\text { ASCT. } \\
\text { Matrices de } \\
\text { Raven. }\end{array}$ & $\begin{array}{l}\text { La evaluación se centró } \\
\text { en la calidad del apego, el } \\
\text { rendimiento cognitivo y el } \\
\text { desarrollo físico de estos } \\
\text { niños. }\end{array}$ & $\begin{array}{l}\text { Los desempeños cognitivos y de } \\
\text { desarrollo son más importantes } \\
\text { en el NIG que en el CAUG. } \\
\text { El rendimiento promedio en } \\
\text { CAUG fue menor que en el NIG. }\end{array}$ \\
\hline
\end{tabular}




\begin{tabular}{|c|c|c|c|c|c|}
\hline Autor, año y título & Objetivo & Muestra & $\begin{array}{l}\text { Instrumentos } \\
\text { de evaluación }\end{array}$ & Procedimiento & Resultados \\
\hline $\begin{array}{l}\text { Levin, Fox, Zeanah, \& } \\
\text { Nelson, (2015) } \\
\text { Social Communication } \\
\text { Difficulties and Autism in } \\
\text { Previously Institutionali- } \\
\text { zed Children. }\end{array}$ & $\begin{array}{l}\text { Determinar el riesgo de difi- } \\
\text { cultades con la comunicación } \\
\text { social y los comportamientos } \\
\text { restringidos/repetitivos, así } \\
\text { como la tasa de TEA en niños } \\
\text { institucionalizados en la } \\
\text { infancia temprana y evaluar el } \\
\text { impacto de una intervención } \\
\text { de acogida temporal para } \\
\text { mejorar este riesgo. }\end{array}$ & $\begin{array}{l}53 \text { niños CAUG, } \\
52 \text { niños FCG y } 96 \\
\text { niños NIG. } \\
\text { De } 8 \text { a } 11 \text { años. }\end{array}$ & $\begin{array}{l}\text { SCQ. } \\
\text { SSRS. } \\
\text { WISC-IV. }\end{array}$ & $\begin{array}{l}\text { Con el SCQ se evaluaron } \\
\text { diferentes ítems: comunica- } \\
\text { ción social, sintomatología } \\
\text { asociada con el TEA. Se } \\
\text { correlaciona con el SSRS, } \\
\text { que valora las habilidades } \\
\text { sociales, los problemas de } \\
\text { comportamiento y la com- } \\
\text { petencia académica. }\end{array}$ & $\begin{array}{l}\text { Los resultados del SCQ mues- } \\
\text { tran que los niños EIG presentan } \\
\text { puntuaciones más altas (más } \\
\text { problemas) que los NIG. } \\
\text { Desempeño del CAUG en el } \\
\text { SCQ fue peor que en el FCG. }\end{array}$ \\
\hline
\end{tabular}

\section{Bick, Fox, Zeanah, \& Nel-} son (2017)

Early deprivation, atypical brain development, and internalizing symptoms in late childhood.
Examinar si las alteraciones

inducidas por el abandono en las zonas seleccionadas de la materia blanca límbica y fronto-estriada y el cuerpo calloso se asociarían con el riesgo competidor y prospectivo de internalización entre los niños criados institucionalmente en el BEIP.
69 participantes:

26 niños CAUG

23 niños FCG y 20 DTI.

niños NIG.

De 8 a 10 años.
Se evaluó la materia blanca utilizando el DTI cuando los niños tenían entre 8 y 10 años. Los síntomas de internalización se evaluaron en el momento de la resonancia magnética.
A los 8 años, CAUG Y FCG tenían más síntomas de ansiedad y depresión que los NIG.

A los 12 años, CAUG más síntomas de depresión y ansiedad que los FCG y NIG. 


\begin{tabular}{|c|c|c|c|c|c|}
\hline Autor, año y título & Objetivo & Muestra & $\begin{array}{l}\text { Instrumentos } \\
\text { de evaluación }\end{array}$ & Procedimiento & Resultados \\
\hline $\begin{array}{l}\text { Humphreys et al., (2015) } \\
\text { Effects of institutional } \\
\text { rearing and foster care } \\
\text { on psychopathology at } \\
\text { age } 12 \text { years in Romania: } \\
\text { follow-up of an open, } \\
\text { randomised controlled } \\
\text { trial. }\end{array}$ & $\begin{array}{l}\text { Evaluar la psicopatología en } \\
\text { niños que habían experimen- } \\
\text { tado crianza institucional en } \\
\text { comparación con niños que } \\
\text { nunca habían estado en una } \\
\text { institución. } \\
\text { Evaluar la efectividad de la } \\
\text { intervención de acogida tem- } \\
\text { poral en la psicopatología en } \\
\text { la adolescencia temprana. } \\
\text { Examinar el potencial de aso- } \\
\text { ciación de la colocación esta- } \\
\text { ble con la psicopatología. }\end{array}$ & $\begin{array}{l}55 \text { niños CAUG, } \\
54 \text { niños FCG ( } 26 \\
\text { niños en un lugar } \\
\text { estable y } 28 \text { en } \\
\text { acogidas interrum- } \\
\text { pidas) y } \\
49 \text { niños NIG. } \\
12 \text { años. }\end{array}$ & DISC-IV. & $\begin{array}{l}\text { Se evaluó con el de DISC- } \\
\text { IV tres dominios de psi- } \\
\text { copatología: síntomas de } \\
\text { internalización, síntomas de } \\
\text { externalización y TDAH. Las } \\
\text { puntuaciones obtenidas se } \\
\text { compararon entre los dife- } \\
\text { rentes grupos. }\end{array}$ & $\begin{array}{l}\text { CAUG y FCG más síntomas de } \\
\text { internalización, externalización } \\
\text { y TDAH que el NIG. } \\
\text { CAUG más síntomas de externa- } \\
\text { lización que FCG interrumpido } \\
\text { y NIG. } \\
\text { CAUG y FCG interrumpidos } \\
\text { más síntomas de internalización } \\
\text { y externalización que los FCG } \\
\text { estables y NIG. }\end{array}$ \\
\hline $\begin{array}{l}\text { Wade, Fox, Zeanah, \& } \\
\text { Nelson, (2019). } \\
\text { Long-term effects of insti- } \\
\text { tutional rearing, foster } \\
\text { care, and brain activity on } \\
\text { memory and executive } \\
\text { functioning }\end{array}$ & $\begin{array}{l}\text { Examinar las trayectorias de } \\
\text { la memoria y la FE desde la } \\
\text { infancia media hasta la adoles- } \\
\text { cencia entre los niños con his- } \\
\text { torias de crianza institucional }\end{array}$ & $\begin{array}{l}47 \text { niños CAUG } \\
52 \text { niños FCG } \\
62 \text { niños NIG } \\
8-16 \text { años. }\end{array}$ & CANTAB. & $\begin{array}{l}\text { Las subpruebas selecciona- } \\
\text { das del CANTAB se adminis- } \\
\text { traron a los niños a los } 8,12 \\
\text { y } 16 \text { años. }\end{array}$ & $\begin{array}{l}\text { En las medidas de atención, } \\
\text { memoria visual a corto plazo y } \\
\text { memoria de trabajo espacial el } \\
\text { NIG tuvo un mayor porcentaje } \\
\text { correcto y menor probabilidad } \\
\text { de error que los CAUG Y FCG. }\end{array}$ \\
\hline $\begin{array}{l}\text { tituciolalized group (grupc } \\
\text { WISC-IV: Escala de intelige } \\
\text { de niños siempre institucic } \\
\text { rio de comunicación socia } \\
\text { co para niños; TDAH: Tras }\end{array}$ & $\begin{array}{l}\text { cia de Wechsler para niños; FE: } \\
\text { alizados); SI: Siempre institucio } \\
\text { rno proyecto de intervención } \\
\text { rnéficit de atención e hip }\end{array}$ & $\begin{array}{l}\text { en cuidado institucic } \\
\text { o); HBQ: Cuestionari } \\
\text { Función ejecutiva; BC } \\
\text { nalizados; ASCT: Tare } \\
\text { Temprada de Bucares } \\
\text { teractividad; CANTAB }\end{array}$ & $\begin{array}{l}\text { nal); FCG: Foster c } \\
\text { de salud y compc } \\
\text { T2-SF: Bruininks-O } \\
\text { final de la historia } \\
\text {; DTI: escaneo del } \\
\text { : Cambridge Neur }\end{array}$ & $\begin{array}{l}\text { e group (grupo de niños de ac } \\
\text { amiento; SSRS: Sistema de eva } \\
\text { eretsky, versión corta; EIG: eve } \\
\text { el apego; TEA: Trastorno del e } \\
\text { ensor de difusión; DISC-IV: Pro } \\
\text { sychological Test Automated }\end{array}$ & $\begin{array}{l}\text { gida temporal); NIG: Never ins- } \\
\text { luación de habilidades sociales; } \\
\text { institucionalized group (grupo } \\
\text { pectro autista; SCQ: Cuestiona- } \\
\text { grama de entrevista de diagnósti- } \\
\text { atery. }\end{array}$ \\
\hline
\end{tabular}


En la Tabla 2 se muestran los estudios realizados con niños adoptados. Todos coinciden en que estos niños presentan déficits en algún aspecto de su desarrollo.

TABLA 2. RESUMEN DE LOS ARTÍCULOS ANALIZADOS DE NIÑOS ADOPTADOS

\begin{tabular}{|c|c|c|c|c|c|}
\hline Autor, año y título & Objetivo & Muestra & $\begin{array}{l}\text { Instrumentos de } \\
\text { evaluación }\end{array}$ & Procedimiento & Resultados \\
\hline $\begin{array}{l}\text { Purvis, McKenzie, } \\
\text { Cross, \& Razuri, } \\
\text { (2013) } \\
\text { A Spontaneous Emer- } \\
\text { gence of Attachment } \\
\text { Behavior in At-Risk } \\
\text { Children and a Cor- } \\
\text { relation with Sensory } \\
\text { Deficits. }\end{array}$ & $\begin{array}{l}\text { Realizar un campa- } \\
\text { mento diurno rico en } \\
\text { estímulos para cono- } \\
\text { cer el efecto de este } \\
\text { en sesiones guiadas } \\
\text { por profesionales en } \\
\text { diferentes mediciones } \\
\text { de niños con proble- } \\
\text { mas de deprivación. }\end{array}$ & $\begin{array}{l}18 \text { partici- } \\
\text { pantes de } \\
3-14 \text { años. }\end{array}$ & $\begin{array}{l}\text { Entrevista a los } \\
\text { padres. } \\
\text { Dibujos de los } \\
\text { niños. } \\
\text { Examen sensorial } \\
\text { administrado por } \\
\text { los TO. } \\
\text { BB. } \\
\text { CBCL. } \\
\text { RADQ. } \\
\text { SQP. }\end{array}$ & $\begin{array}{l}\text { Se realizaron campamentos } \\
\text { diurnos terapéuticos durante } 3 \\
\text { semanas para niños en riesgo, } \\
\text { para determinar si las terapias } \\
\text { multimodales podrían mejorar } \\
\text { los efectos de un traumatismo } \\
\text { complejo del desarrollo. }\end{array}$ & $\begin{array}{l}\text { TO indican que muchos niños empiezan el } \\
\text { campamento con déficits en: Coordinación } \\
\text { mano-ojo, propiocepción, vestibular y táctil. } \\
\text { Los padres inciden la presencia de déficits } \\
\text { sensoriales: auditivos, visuales, táctiles, olfa- } \\
\text { torios, vestibulares, de coordinación y en el } \\
\text { tono muscular. } \\
\text { Existe correlación entre el SQP y las evalua- } \\
\text { ciones realizadas por los TO de la función } \\
\text { vestibular y comportamiento de apego. }\end{array}$ \\
\hline $\begin{array}{l}\text { Eckerle et al., (2014) } \\
\text { Vision and Hearing } \\
\text { Deficits and Asso- } \\
\text { ciations with Parent- } \\
\text { Reported Behavioral } \\
\text { and Developmental } \\
\text { Problems in Interna- } \\
\text { tional Adoptees. }\end{array}$ & $\begin{array}{l}\text { Determinar los défi- } \\
\text { cits de visión y audi- } \\
\text { ción en adoptados } \\
\text { internacionales. } \\
\text { y sus asociaciones con } \\
\text { problemas emocio- } \\
\text { nales, conductuales y } \\
\text { cognitivos. }\end{array}$ & $\begin{array}{l}1005 \text { niños } \\
\text { de entre } \\
\text { 4-18 años. }\end{array}$ & $\begin{array}{l}\text { Entrevista/encues- } \\
\text { ta a los padres. } \\
\text { CBCL. } \\
\text { HVS. }\end{array}$ & $\begin{array}{l}\text { A los padres de niños adopta- } \\
\text { dos se les pasó una encuesta } \\
\text { (con preguntas sobre las expe- } \\
\text { riencias previas a la adopción } \\
\text { del niño y los diagnósticos } \\
\text { médicos posteriores) y la CBCL. }\end{array}$ & $\begin{array}{l}\text { A través del HVS se detectó que el } 31 \% \text { de } \\
\text { los niños presentaban problemas de visión y } \\
\text { el } 12,8 \% \text { problemas de audición. } \\
\text { A través de MnIAP se detectó que el } 24,7 \% \\
\text { tenía problemas de visión y el } 11,9 \% \text { proble- } \\
\text { mas de audición. }\end{array}$ \\
\hline
\end{tabular}




\begin{tabular}{|c|c|c|c|c|c|}
\hline Autor, año y título & Objetivo & Muestra & $\begin{array}{l}\text { Instrumentos de } \\
\text { evaluación }\end{array}$ & Procedimiento & Resultados \\
\hline $\begin{array}{l}\text { Elovainio, Raaska, } \\
\text { Sinkkonen, \& Akip, } \\
\text { (2015) } \\
\text { Associations between } \\
\text { attachment-related } \\
\text { symptoms and } \\
\text { later psychological } \\
\text { problems among } \\
\text { international adop- } \\
\text { tees: Results from the } \\
\text { FinAdo study. }\end{array}$ & $\begin{array}{l}\text { Examinar si los sínto- } \\
\text { mas relacionados con } \\
\text { el apego estaban aso- } \\
\text { ciados con problemas } \\
\text { conductuales / emo- } \\
\text { cionales posteriores y } \\
\text { los síntomas de TDAH. }\end{array}$ & $\begin{array}{l}591 \text { niñas y } \\
768 \text { niños de } \\
\text { entre } 6-15 \\
\text { años. }\end{array}$ & $\begin{array}{l}\mathrm{CBCL} \\
\mathrm{KCAQ}\end{array}$ & $\begin{array}{l}\text { Se examinaron las asociaciones } \\
\text { entre los síntomas relacionados } \\
\text { con el apego y problemas psi- } \\
\text { cológicos posteriores entre los } \\
\text { adoptados internacionales. } \\
\text { Los cuestionarios fueron pasa- } \\
\text { dos a los padres adoptivos y a } \\
\text { los niños mayores de } 9 \text { años. }\end{array}$ & $\begin{array}{l}\text { Niños con problemas de apego tenían más } \\
\text { problemas de comportamiento, de interna- } \\
\text { lización, de externalización y mas síntomas } \\
\text { de TDAH. }\end{array}$ \\
\hline
\end{tabular}

\footnotetext{
FinAdo study.
}

\section{Examinar si la región}

Roeber, Gunnar, \& Pollak, (2014)

Early Deprivation

Impairs the Development of Balance and

Bilateral Coordination. de nacimiento moderaba los resultados claves para conocer eras específicos de la

Rita et al., (2017)

Child and family-related predictors of psychological outcomes in children adopted from abroad; what is the role of caregiver time?

\section{2 niños: 48 $\mathrm{Pl}, 40$ FCy 44 niños no \\ BOTMP.} adoptados. Edad: 8 y 9 años. si los efectos de la atención institucional región de nacimiento del niño.

Determinar cuáles de los síntomas clínicos tempranos del niño podrían servir como signos tempranos de advertencia para problemas posteriore agrupándolos en cuatro categorías.

\section{5 adop-} tados, 708 niñas y 557 niños.

Edad media:

2 años $(0,1$ a 6,9 años).
Se administraron individualmente a los niños, las subpruebas de equilibrio y coordinación bilateral del BOTMP
PI y FC tuvieron puntuaciones más bajas en el equilibrio que el NIG.

PI tuvo puntuaciones más bajas en la coordinación bilateral que el FC y NIG.

TO: Terapia Ocupacional; BB: Lista de verificación del trastorno de apego de Beech Brook; CBCL: Lista de verificación de la conducta del niño; RADQ: Cuestionario de trastorno de apego de Randolph; SQP: Cuestionario de historia sensoriomotora para padres; HVS: encuesta sobre los problemas de visión y audición; Mnlap: The Minnesota International Adoption Project; FinAdo: Finish Adoption study; ; TDAH: Trastorno por déficit de atención e hiperactividad; KCAQ: Cuestionario de apego de la familia central; PI:Posti-nstitucionalizados; FC: Foster Care (acogida temporal); BOTMP: Prueba Bruininks-Oseretsky del dominio Motor) ;NIG: Never instituciolalized group (grupo de niños nunca institucionalizados); FTF: Escala de cinco a quince. 


\section{Discusıón}

El objetivo del trabajo ha sido conocer el estado actual de las dificultades de IS en niños huérfanos, institucionalizados y adoptados, ya que, en ocasiones, dichos problemas no son evidentes. En los trabajos de Purvis et al. (2013), Rita et al., (2017) y Wade et al., (2019) se indica que los déficits presentados en los niños institucionalizados o adoptados se correlacionarían positivamente con problemas en el procesamiento sensorial, ya que los niños presentan déficits de coordinación, propiocepción, vestibulares, táctiles, auditivo y olfatorios, y en general presentan déficit en la función ejecutiva. La mayoría de los artículos analizados evalúan a través de diversas escalas el desarrollo y la conducta del niño, mostrando mayores dificultades los niños institucionalizados que los que no lo han estado nunca (Elovainio et al., 2015; McDermott et al., 2013). Estas dificultades son, en ocasiones, explicadas por un trastorno por déficit de atención e hiperactividad (Humphreys et al., 2015), por dificultades en las funciones cognitivas como son atención, memoria visual, memoria a corto plazo y de trabajo (Mbiya Muadi et al., 2014; Wade et al., 2019) problemas en la en la comunicación y habilidades sociales (Levin et al., 2015) y/o por sintomatología psiquiátrica, incluyendo ansiedad y depresión (Bick et al., 2017).

Algunos problemas motores son resultado de un inadecuado procesamiento sensorial (Miller et al., 2007). En los trabajos de Levin et al., (2014); Purvis et al., (2013) y Roeber et al. (2014) los niños institucionalizados y adoptados obtienen puntuaciones inferiores en el área motora, destacando la coordinación y el equilibrio.

En este trabajo se han producido limitaciones. Entre ellas, quizá sea necesario prestar mayor atención a la selección de las palabras clave, así como la realización de una búsqueda más amplia, consultando en otras bases de datos. Como líneas futuras se plantean la realización de una revisión sistemática mejorando la calidad metodológica del estudio, así como contemplar otras metodologías que utilizan los terapeutas ocupacionales, además de la IS. Otra línea interesante sería evaluar a niños que estén institucionalizados en nuestro país, así como a niños acogidos y/o adoptados, para así poder desarrollar intervenciones eficaces que mejoren su desempeño ocupacional y la calidad de vida familiar.

\section{Conclusiones}

A la luz de los estudios encontrados, parece ser que los niños institucionalizados, huérfanos y adoptados presentan dificultades en su desarrollo y en su salud. Algunas de las dificultades analizadas podrían estar relacionadas con problemas en la IS.

Creemos que este trabajo puede ser un punto de partida para comenzar a recabar información sobre cómo desde Terapia Ocupacional en general, y desde el enfoque de IS en particular, se podría apoyar el desempeño ocupacional de estos niños.

\section{RefERENCIAS BibLIOGRÁFICAS}

Anaby, D., Law, M., Teplicky, R., \& Turner, L. (2015). Focusing on the Environment to Improve Youth Participation: Experiences and Perspectives of Occupational Therapists. International Journal of Environmental Research and Public Health, 12(10), 13388-13398. https://doi.org/10.3390/ijerph121013388

AOTA. (2014). Occupational therapy practice framework: Domain and process ( $3^{\mathrm{a}}$ ed.). American Journal of Occupational Therapy, 68(supl. 1), S48.

Ayres, J., \& Robbins, J. (2008). La Integración sensorial en los niños: Desafíos sensoriales ocultos. Madrid: TEA ediciones.

Ayres, J. (1972). Sensory integration and learning disorders. Los Ángeles: Western Psychological Services.

Bick, J., Fox, N., Zeanah, C., \& Nelson, C. A. (2017). Early deprivation, atypical brain development, and internalizing symptoms in late childhood. Neuroscience, 342, 140-153. https://doi.org/10.1016/j. neuroscience.2015.09.026

Cermak, S. (2009). Deprivation and Sensory Processing in Institutionalized and Postinstitutionalized Children: Part I. Special Interest Section Quarterly, 32(2), 1-4.

Chamorro Noceda, L. A. (2012). El apego. Su importancia para el pediatra. Pediatría (Asunción), 39(3), 199-206.

del Moral Orro, G., Pastor Montaño, M. A., \& Sanz Valer, P. (2013). Del Marco Teórico de Integracion Sensorial al Modelo Clinico de Intervención. Revista de Terapia Ocupacional de Galicia, 10(17), 1-25.

Domenech, E. (2001). Trastornos emocionales y patologías del lenguaje. En J. Narbona \& C. Chevrie-Mulle, Desarrollo normal, evaluación y trastornos (Narbona y C. Chevrie-Mulle, pp. 383-392). Barcelona: Masson.

Domínguez Jiménez, I., \& Calvo Arenillas, J. ignacio. (2015). Terapia Ocupacional y su papel en Atención Temprana: Revisión sistemática. Revista de Terapia Ocupacional de Galicia, 12(21), 1-22. 
Eckerle, J. K., Knauf Hill, L., Inverson, S., Hellerstedt, W., Gunnar, M., \& Johnson, D. (2014). Vision and Hearing Deficits and Associations with Parent-Reported Behavioral and Developmental Problems in International Adoptees. Matern Child Health, 18(3), 575-583. https://doi.org/10.1007/s10995-013-1274-1.Vision

Elovainio, M., Raaska, H., Sinkkonen, J., \& Akip, S. M. (2015). Associations between attachment-related symptoms and later psychological problems among international adoptees: Results from the FinAdo study. Scandinavian Journal of Psychology, 56(1), 53-61. https:// doi.org/10.1111/sjop.12174

Federación Estatal de Asociaciones de Profesionales de Atención Temprana. (2005). Libro blanco de la Atención Temprana. Madrid: Real Patronato sobre Discapacidad.

Hernández-muela, S., Mulas, F., Meneses, M. T.D., \& Roselló, B. (2003). Niños adoptados: Factores de riesgo y problemática neuropsicológica. 36(Supl 1), 108-117.

Humphreys, K. L., Gleason, M. M., Drury, S.S., Miron, D., Nelson, C. A., Fox, N. A., \& Zeanah, C.H. (2015). Effects of institutional rearing and foster care on psychopathology at age 12 years in Romania: Follow-up of an open, randomised controlled trial. The Lancet Psychiatry, 2(7), 625-634. https://doi.org/10.1016/ S2215-0366(15)00095-4

Juffer, F., van ljzendoorn, M. H., \& Palacios, J. (2011). Recuperación de niños y niñas tras su adopción. Infancia y Aprendizaje, 34(1), 3-18. https://doi.org/10.1174/021037011794390102

Levin, A. R., Fox, N. A., Zeanah, C. H., \& Nelson, C. A. (2015). Social Communication Difficulties and Autism in Previously Institutionalized Children. Journal of the American Academy of Child \& Adolescent Psychiatry, 54(2), 108-115. el. https://doi.org/10.1016/j. jaac.2014.11.011

Levin, A. R., Zeanah, C. H., Fox, N. A., \& Nelson, C. A. (2014). Motor Outcomes in Children Exposed to Early Psychosocial Deprivation. The Journal of Pediatrics, 164(1), 123-129.e1. https://doi. org/10.1016/j.jpeds.2013.09.026

Mbiya Muadi, F., Mampunza, S., Symann, S., Habimana, L., D'Hoore, W., Malengreau, M., ... Charlier-Mikolajczak, D. (2014). Attachement et développement des enfants abandonnés vivant en institution résidentielle à Kinshasa. Archives de Pédiatrie, 27(11), 1159-1166. https://doi.org/10.1016/j.arcped.2014.08.037

McDermott, J.M., Troller-Renfree, S., Vanderwert, R., Nelson, C. A., Zeanah, C.H., \& Fox, N.A. (2013). Psychosocial deprivation, executive functions, and the emergence of socio-emotional behavior problems. Frontiers in Human Neuroscience, 7. https:// doi.org/10.3389/fnhum.2013.00167

Miller, L. J., Anzalone, M. E., Lane, S. J., Cermak, S. A., \& Osten, E. T. (2007). Concept evolution in sensory integration: A proposed nosology for diagnosis. American Journal of Occupational Therapy, 67(135), 135-140.

Observatorio de la Infancia. (2018). Boletín de datos estadísticos de medidas de protección a la infancia: Datos 2017 (Dirección General de Servicios para la Familia y la Infancia. Ministerio de Sanidad, Servicios Sociales e Igualdad). Madrid: Boletín Estadístico; 20.

Observatorio para la Confianza. (2019). Informe 2018: Situación de Derechos Humanos de los niños, niñas y adolescentes bajo protección del Estado de Chile. Providencia.

Papalia, D. E., Martorrell, G., \& Duskin Feldman, R. (2013). Desarrollo

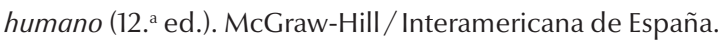

Petrowski, N., Cappa, C., \& Gross, P. (2017). Estimating the number of children in formal alternative care: Challenges and results. Child Abuse \& Neglect, 70, 388-398. https://doi.org/10.1016/j. chiabu.2016.11.026

Purvis, K. B., McKenzie, L. B., Cross, D. R., \& Razuri, E. B. (2013). A Spontaneous Emergence of Attachment Behavior in At-Risk Children and a Correlation With Sensory Deficits: A Spontaneous Emergence of Attachment Behavior in At-Risk Children and a Correlation With Sensory Deficits. Journal of Child and Adolescent Psychiatric Nursing, 26(3), 165-172. https://doi.org/10.1111/jcap.12041

Rajmil, L. (2013). La crisis económica afecta la salud infantil: ¿qué papel tienen los pediatras? Anales de Pediatría, 79(4), 205-207. https:// doi.org/10.1016/j.anpedi.2013.05.028

Rita, N., Elovainio, M., Raaska, H., Lahti-Nuuttila, P., Matomäki, J., Sinkkonen, J., \& Lapinleimu, H. (2017). Child and family-related predictors of psychological outcomes in children adopted from abroad; what is the role of caregiver time? Scandinavian Journal of Psychology, 58(4), 312-317. https://doi.org/10.1111/sjop.12374

Roeber, B. J., Gunnar, M. R., \& Pollak, S. D. (2014). Early deprivation impairs the development of balance and bilateral coordination: Deprivation and Motor Development. Developmental Psychobiology, 56(5), 1110-1118. https://doi.org/10.1002/dev.21159

Servicio Nacional de Menores. (2019). Balance de Gestión Integral año 2018. Santiago Centro: Ministerio de Justicia y Derechos Humanos.

UNICEF. (2015). Huérfanos. Recuperado de https://www.unicef.org/ es/huérfanos

Vega-Arce, M., \& Nuñez-Ulloa, G. (2017). Experiencias Adversas en la Infancia: Revisión de su impacto en niños de 0 a 5 años. Enfermerí universitaria, 14, 124-130.

Wade, M., Fox, N. A., Zeanah, C. H., \& Nelson, C. A. (2019). Long-term effects of institutional rearing, foster care, and brain activity on memory and executive functioning. Proceedings of the National Academy of Sciences, 116(5), 1808-1813. https://doi.org/10.1073/ pnas.1809145116

Wiik, K. L., Loman, M.M., Ryzin, M. J. V., Armstrong, J.M., Marilyn, J., Pollak, S. D., \& Gunnar, M. R. (2011). Behavioral and Emotional Symptoms of Post-Institutionalized Children in Middle Childhood. Journal of Child Psychology and Psychiatry, 52(1), 56-63. https:// doi.org/10.1111/j.1469-7610.2010.02294.x.Behavioral 